\title{
Arterial occlusion mimicking vasculitis in a patient with incontinentia pigmenti
}

\author{
E. Beccastrini - G. Baldereschi - M. M. D'Elios • \\ L. Emmi
}

Received: 11 August 2012/Accepted: 9 February 2013/Published online: 1 March 2013

(C) Springer-Verlag Italia 2013

\begin{abstract}
A large number of diseases can mimic a vasculitis. The diagnosis can be challenging due to the similarity with several diseases that have a different pathogenesis. As reported in the literature, incontinentia pigmenti (IP), a rare genetic disorder, can present vascular alterations on eye, brain and lung. We report a case of peripheral arterial disease in a patient with IP, suggesting further vascular localizations of the disease.
\end{abstract}

Keywords Vasculitis - Incontinentia pigmenti ·

Peripheral arterial disease $\cdot$ Skin ulcer

Incontinentia pigmenti (IP), or Bloch-Sulzberger syndrome, is a rare $\mathrm{X}$-linked genetic disorder ascribed to the NF-kB essential modulator (NEMO)/IkB kinase- $\gamma$ $(\mathrm{IKBK} \gamma)$ gene mutation. The NF-kB signaling pathway regulates the expressions of genes that are involved in many immune, inflammatory and apoptotic mechanisms [1]. IP has a wide spectrum of clinical presentations. Skin lesions classically evolve through four stages: a vescicolobollous, verrucous, hyperpigmented and finally, a hypopigmented stage usually lasting a lifetime. Alopecia, nail, breast, dental, ocular or central nervous system abnormalities can also be observed. The diagnosis is principally based on clinical criteria (Table 1) and molecular genetic

E. Beccastrini · M. M. D’Elios · L. Emmi ( $₫)$

Department of Biomedicine, Patologia Medica, Center for Systemic Autoimmune Diseases, Lupus Clinic, Careggi University Hospital, L.go Brambilla 3, 50134 Florence, Italy e-mail: lorenzoemmi@yahoo.it

G. Baldereschi

Department of Heart and Vessels, Careggi University Hospital, Florence, Italy testing of NEMO mutation. In the majority of cases, IP is caused by a large-scale deletion of NEMO exons $4-10$ $[2,3]$.

Retinal and brain vascular abnormalities are well described in the literature $[4,5]$. We report a case of peripheral arterial disease in a young patient with IP.

A 16-year-old female was referred to our centre because of a suspected vasculitis. From her childhood she had presented bilateral coldness and slight cyanosis of the lower extremities. In the last years, however, cyanosis and coldness progressively worsened, with the appearance of severe pain of the toes. At the same time, skin ulcers appeared on the first and fourth left toes (Fig. 1a, b).

A detailed analysis of the clinical history gave no evidence of autoimmune diseases, vasculitis or coagulation defects. Laboratory tests, such as full blood count, metabolic panel, erythrocyte sedimentation rate (ESR) and C-reactive protein (CRP) were within normal limits. Antinuclear antibodies (ANA), anti-dsDNA, anti-extractable nuclear antigens (ENA), anti-neutrophil cytoplasm antibodies (ANCA), anti-phospholipid antibodies (aPL) and lupus anticoagulant (LAC) were absent. Repeated evaluations of serum specimens, collected and separated while warm, were negative for cryoglobulins, complement fractions (C3, C4) and rheumatoid factor (RF). Other coldrelated diseases were also excluded by specific tests, such as cold agglutinins or cryofibrinogen. Doppler ultrasound (US) examination of the lower extremities gave findings compatible with a complete bilateral occlusion of the posterior tibial artery with a collateral circulation maintaining a reduced blood flow below the occlusion. The US also revealed a severe bilateral stenosis of the anterior tibial artery. Complete US evaluation of the other arteries and an echocardiogram were normal. The 18F-fluorodeoxyglucose positron emission tomography (PET) did not 
Table 1 Diagnostic criteria for IP

Major criteria
Erythema followed by blisters/vesicles
Skin involvement stage I (first weeks of life to age four months)
Hyperpigmented streaks and whorls that respects
Blaschko's lines
Skin involvement stage III (age four months to 16 years)
Pale, hairless, atrophic linear streaks or patches
Skin involvement stage IV (adolescence through adulthood)
Minor criteria
Hypodontia, anodontia, microdontia, and abnormally shaped teeth
Alopecia
Mild ridging or pitting of the nails, Onychogryphosis
Peripheral neovascularization of the retina

The diagnosis can be made if at least one of the major criteria is present

document local metabolic activity nor gave evidence of systemic underlying disease. Magnetic resonance angiography (MRA) showed progressive narrowing of the tibial artery and its apparent absence in the distal tract, but was early interrupted because of an adverse reaction to the contrast agent. The angiogram of the lower extremities, however, confirmed the lack of blood flow to tibial arteries (Fig. 1c, d).

The patient did not give the consent to undergo a skin biopsy. She had no risk factors for vascular disease, but she had a 1-pack year history, having started smoking at the age of 14. Therefore, a Buerger's disease was suspected. The patient gave up smoking and started steroid therapy but did not show any improvement. Due to the lack of response and the absence of thrombotic and inflammatory findings, other diagnostic hypotheses were evaluated. The patient is the only child of non-consanguineous caucasian parents and her mother was affected by IP. At birth, the patient had presented an erythematous rash followed by some vescicle eruptions with a linear pattern on forearms and thigh, and IP had been suspected. At present, a complete skin examination disclosed slight hypopigmented areas of both legs and dystrophic changes in toenails. The patient did not present dental or ocular abnormalities and the brain magnetic resonance was negative. The deletion of exons 4-10 within the NEMO gene was found, hence confirming the diagnosis of IP.

Our findings support the potential relevance of vascular pathology in IP, by demonstrating vascularization defects of the lower extremities in a patient with IP. It was not possible to obtain further evidence of such an association through biopsy specimens or other diagnostic tools. Skin biopsy was not taken, because the patient refused consent but, keeping in mind the presence of the arterial obstruction, we did not expect local alterations of circulation or skin. Repeated controls, however, seem to exclude other potential diagnosis. Buerger's disease was excluded based on several clinical and instrumental findings. First,
Fig. 1 Ulcers of the left foot at an early stage $(\mathbf{a}, \mathbf{b})$. Angiogram of lower extremities showing bilateral interruption of the tibial arteries $(\mathbf{c}, \mathbf{d})$
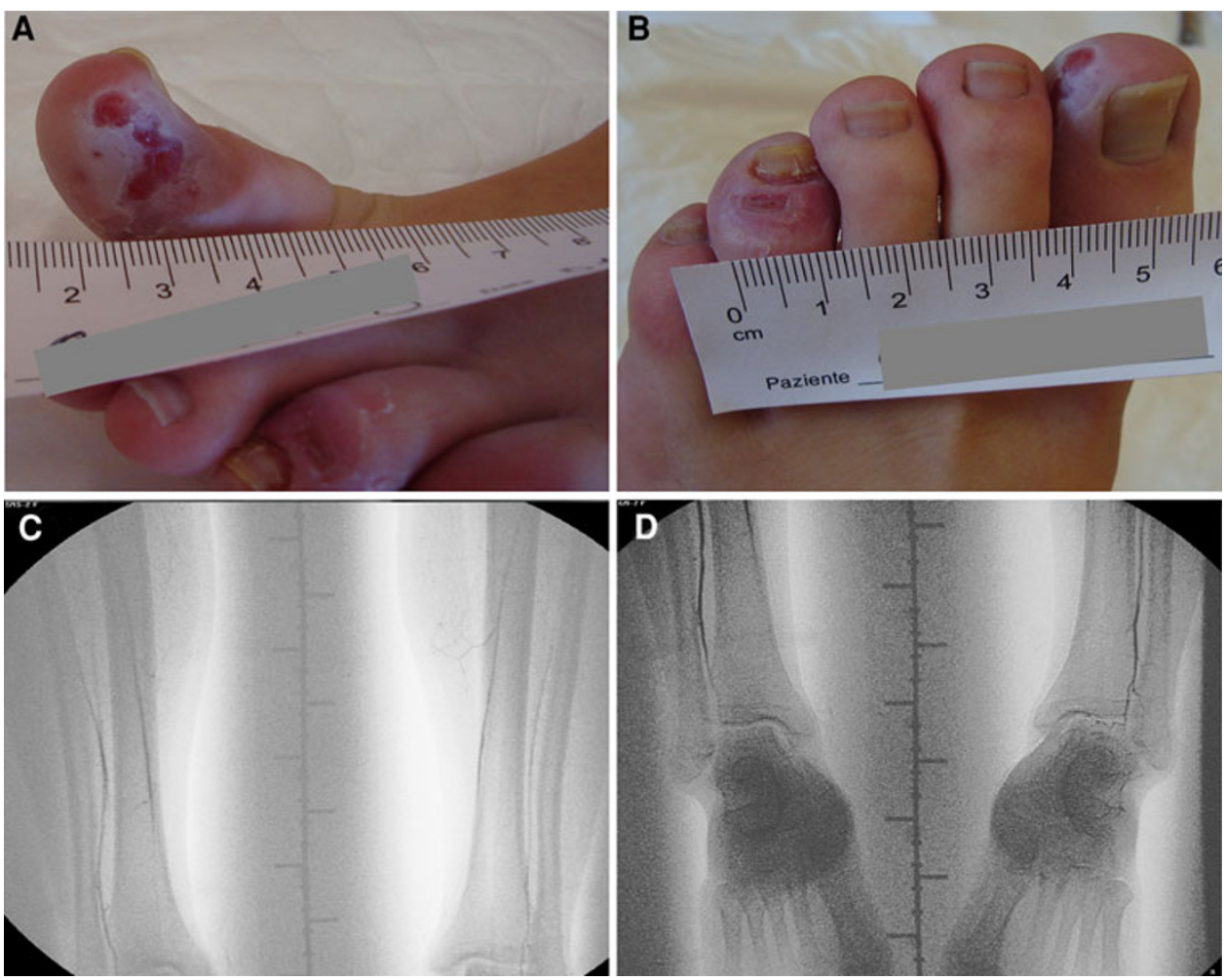
coldness and cyanosis of the lower extremities have appeared long before the patient started to smoke and she was not heavy smoker. Second, instrumental findings revealed major defects in the blood flow, without signs of inflammation or thrombosis. Chilblain lupus was considered, but the presence of arterial obstructions did not fit with such a diagnosis. A diagnosis of fibromuscolar dysplasia (FMD) was also considered. FMD, a degenerative angiopathy of the medium-sized arteries, usually affects young females and can virtually affect every arterial district. A complete evaluation of the arterial tree, however, did not reveal alterations of the arterial districts that are most frequently involved in FMD, such as renal and carotid ones. Although literature reports the involvement of extremities in FMD, in our case angiographic findings did not show the typical "string of beads" aspect and there was no clinical or instrumental evidence suggesting FMD.

On the other hand, the importance of vasculopathy as a pathogenetic mechanism in IP is supported by several studies [6-8]. The majority of ocular changes observed in IP can arise from retinal vascular abnormalities and the central nervous system dysfunctions can be associated with microangiopathic processes or arterial defects [9]. Finally, three cases of pulmonary hypertension associated to vascular lung abnormalities have been described to date [10]. NEMO gene mutation plays a critical role in anti-apoptotic processes. NF-kB reduced activity was associated with a wide spectrum of defects, involving many different organs. As suggested by the literature data, through unknown mechanisms, vasculopathy can be a significant feature of the IP phenotype, potentially related to altered apoptotic pathways. We can hypothesize an abnormal development of the arterial wall with a functional component. The abnormalities in vascular development observed in IP patients on brain, eye and lung lead to ischemic attacks, strokes, retinal detachment and arterial hypertension, respectively. Although the pathogenetic mechanism is still elusive, we can think that also the peripheral arteries can present similar abnormalities in vascular development, inducing a critical reduction in the blood flow. Such an involvement, not yet reported in the literature, cannot be demonstrated through specific tests to date and should be confirmed by further observations. Our report, however, could suggest the usefulness of carefully collecting the medical history in young patients with suspected vasculitis, considering IP as a possible cause of arterial obstruction.

Conflict of interest E. Beccastrini, G. Baldereschi, M.M. D’Elios, L. Emmi declare that they have no conflict of interest.

Informed Consent All procedures followed were in accordance with the ethical standards of the responsible committee on human experimentation (institutional and national) and with the Helsinki Declaration of 1975, as revised in 2008. Informed consent was obtained from all patients for being included in the study.

Animal studies No animal studies were carried out by the authors for this article.

\section{References}

1. Smahi A, Courtois G, Vabres P et al (2000) Genomic rearrangement in NEMO impairs NF-kB activation and is cause of incontinentia pigmenti. Nature 405:466-472

2. Scheuerle A, Ursini MV (1999) Incontinentia Pigmenti. In: Pagon RA, Bird TD, Dolan CR, Stephens K, Seattle WA (eds) Gene Reviews. University of Washington, Seattle

3. Landy SJ, Donnai D (1993) Incontinentia pigmenti (BlochSulzberger syndrome). J Med Genet 30:53-59

4. Hadj-Rabia S, Rimella A, Smahi A (2011) Clinical and histologic features of incontinentia pigmenti in adults with nuclear factor$\mathrm{kB}$ essential modulator gene mutations. J Am Acad Dermatol 64:508-515

5. Curtois G, Smahi A (2006) NF-kB-related genetic disease. Cell Death Differ 13:843-851

6. Hennel AJ, Ekert PG, Volpe JJ et al (2003) Insight into the pathogenesis of cerebral lesions in incontinentia pigmenti. Pediatr Neurol 29:148-150

7. Fiorillo L, Sinclair DB, Ml O’Byrne et al (2003) Bilateral cerebrovascular accidents in incontinentia pigmenti. Pediatr Neurol 29:66-68

8. Shah SN, Gibbs S, Upton CJ et al (2003) Incontinentia pigmenti associated with cerebral palsy and cerebral leukomalacia: a case report and literature review. Pediatr Dermatol 20:491-494

9. O’Doherty M, Mc Creery K, Green AJ et al (2011) Incontinentia pigmenti-ophthalmological observation of a series of cases and review of the literature. Br J Ophthalmol 95:11-16

10. Hayes IM, Varigos G, Upjohn EJ et al (2005) Unilateral acheiria and fatal pulmonary hypertension in a girl with incontinentia pigmenti. Am J Med Genet A 135:302-303 